\title{
The sequelae of untreated maternal hypothyroidism
}

\author{
M L Mitchell ${ }^{1}$ and R Z Klein ${ }^{2}$ \\ ${ }^{1}$ New England Newborn Screening Program, University of Massachusetts Medical School, 305 South Street, Boston, MA 02130, \\ USA and ${ }^{2}$ Dartmouth Medical School, Lebanon, NH 03756, USA \\ (Correspondence should be addressed to M L Mitchell; Email: marvin.mitchell@umassmed.edu)
}

\begin{abstract}
The concept that gestational subclinical hypothyroidism could have deleterious effects on the intellectual outcome of progeny was championed more than three decades ago by Evelyn Man in a series of publications. Her studies lay fallow until the Spanish group directed by Morreale de Escobar and the Dutch group headed by Vulsma provided the rationale for her results. Although the findings of the Spanish and Dutch groups elucidated the pathophysiologic basis for Man's conclusions, questions remained regarding the reliability of her biochemical measurements and possible bias in patient selection. In view of the uncertainty surrounding the validity of Man's work, we decided to try to confirm her findings. Our initial goal was to obtain an estimate of the prevalence of subclinical hypothyroidism in an unselected population living in New England. We accomplished this with two separate prospective studies involving 12000 pregnant women residing in Maine. We found that $2.3 \%$ had TSH concentrations of $>6.0 \mathrm{mU} / \mathrm{l}$ and $0.3 \%$ had TSH values of $>12 \mathrm{mU} / \mathrm{l}$ at 17 weeks' gestation. We next did a retrospective study, utilizing sera that had been stored at $-20^{\circ} \mathrm{C}$ for 8 years, obtained in week 17 of gestation from 25000 women. We identified 62 women with subclinical hypothyroidism and 124 matched controls. Fourteen of the hypothyroid mothers had been diagnosed and treated before and during pregnancy on a dosage of thyroxine that was unchanged. WISC IQs of the offspring of the 124 control and 62 hypothyroid mothers were determined at $8 \pm 0.5$ years. The mean and S.D. of IQs of the children of the 124 control and of the 14 treated hypothyroid mothers were significantly higher than those of the children of the 48 untreated hypothyroid women. More than twice as many children of the untreated mothers had IQs of $>1$ s.D. below the control mean, and four times as many of the children had IQs 2 S.D. below the control mean, as did the children of the controls. A comparison of the mean hormonal values of the treated and untreated mothers at 17 weeks showed no significant difference in any of the biochemical markers. We surmise that the circulating level of thyroxine was normal in the treated mothers at a critical time before 17 weeks' gestation, but by 17 weeks it was insufficient to meet the growing demands of pregnancy. Treatment should begin as early as possible in pregnancy with the goal of maintaining free thyroxine $\left(\mathrm{FT}_{4}\right)$ in the upper half of the normal reference range and TSH in the lower half of the normal reference range. In view of these data, we believe that all pregnant women should be screened for hypothyroidism as early in pregnancy as possible (or even before conception). To be cost-effective, screening programs should be based on those designed for congenital hypothyroidism, in which filter-paper blood specimens are forwarded to regional laboratories for thyroid function determinations.
\end{abstract}

European Journal of Endocrinology 151 U45-U48

\section{Introduction and background}

Considering the widespread clinical implications of gestational subclinical hypothyroidism and its sequelae, it is unfortunate that it has taken so long for this subject to come to the attention of the medical community. In all likelihood, the reason for the delay can be attributed to the failure to recognize the critical role that thyroxine played (and plays) in maternal-fetal physiology. Some older readers may recall that one of the overriding issues in the early years of screening newborns for congenital hypothyroidism was the normal appearance of the hypothyroid neonate. How could a hypothyroid infant with sky-high TSH and barely detectable thyroxine $\left(\mathrm{T}_{4}\right)$ have no apparent clinical signs of the disorder at birth? Although speculations ranged from the sublime to the ridiculous, maternal thyroxine, when considered, was quickly dismissed because conventional wisdom at that time held that little or no thyroxine crossed the placenta.

Eventually, conventional wisdom was proved to have no basis in fact by the seminal studies in rats by Gabriella Morreale de Escobar and co-workers (1). The Spanish investigators demonstrated that $\mathrm{T}_{4}$ crossed the placenta in sufficient amounts to normalize the triiodothyronine $\left(\mathrm{T}_{3}\right)$ concentration in the brain of the hypothyroid fetal rat. They also showed that hypothyroidism in the pregnant dam caused developmental delay in the offspring. 
Several years later, Vulsma and colleagues demonstrated the importance of the maternal-fetal transfer of $\mathrm{T}_{4}$ in hypothyroid newborns who were incapable of synthesizing $\mathrm{T}_{4}$. Substantial levels of $\mathrm{T}_{4}$ were found in the cord blood of these infants, whose only source of the hormone was from their mothers (2).

The latter studies underscored the importance of maternal $\mathrm{T}_{4}$ in protecting the fetus from the damage of untreated hypothyroidism during gestation. In addition, the results also highlighted the potential adverse effects of gestational hypothyroidism on fetal development before the start of normal fetal thyroid function. Given that fetal thyroid is not fully functional until mid-gestation, it seemed reasonable to ask whether suboptimal concentrations of maternal thyroxine might also have a deleterious effect on an otherwise normal child.

\section{Previous studies}

Interestingly, more than a quarter of a century ago, Evelyn Man, in the first of a series of publications, reported that children of mothers with inadequately treated hypothyroidism had significantly lower IQs than those of adequately treated patients or normal controls (3). Unfortunately, her papers were difficult to understand and to follow. Her studies were also found wanting because the selection process used to identify adequately and inadequately treated patients was unclear. Because of these criticisms, coupled with the belief that little or no thyroxine crossed the placenta, few in the medical community accepted her work.

In view of the potential clinical implications of Man's results, we thought it worthwhile to try to confirm her findings. As an initial step, we determined the prevalence of subclinical hypothyroidism in an unselected population of pregnant women. This was done by measuring thyroid function markers in blood specimens obtained in two separate prospective studies of 12000 women in Maine (USA) who were 17 weeks pregnant. Thyroid function tests showed that $2.3 \%$ of the women had TSH values of $>6.0 \mathrm{mU} / \mathrm{l}, 0.3 \%$ had TSH values of $>12 \mathrm{mU} / \mathrm{l}$ and $70 \%$ of the women with abnormal TSH values had thyroid peroxidase (TPO) antibodies vs $11 \%$ of the controls $(4,5)$. Free $\mathrm{T}_{4}\left(\mathrm{FT}_{4}\right)$ concentrations were 2 S.D.s below the control mean in $0.3 \%$ of the women with the highest TSH concentrations.

These data were instrumental in seeking funding for a large-scale study on the developmental outcome of progeny from women with subclinical hypothyroidism. We decided that a retrospective study using stored aliquots of serum previously collected from women who had been tested for alpha-fetoprotein levels, as were those cited in the above study, would provide results in the shortest time. Needless to say, we were overly optimistic; it took 7 years to complete the work because of difficulties in obtaining financial support.
The study involved measuring TSH in sera that had been collected at 17 weeks' gestation from more than 25000 women in Maine (6). The sera had been stored for approximately 8 years at $-20^{\circ} \mathrm{C}$ before they were retrieved for testing. Women were considered to be hypothyroid if their TSH concentrations were at or above the 97.7 percentile. We identified 62 women with hypothyroidism and matched them with 124 controls whose TSH concentrations were below the 97.7 percentile. Fourteen of the 62 hypothyroid mothers had been diagnosed and treated before and during pregnancy with a dose of thyroxine that remained unchanged.

\section{Effects on offspring}

The children underwent neuropsychological testing at approximately 8 years of age. All had been full term, and none had been hypothyroid at birth or at the time of testing. The children underwent 15 tests, encompassing intelligence, reading, language, attention, school performance and visual motor performance. Although the children of the 62 hypothyroid mothers did less well on the tests than did the children of the controls, their full-scale IQs were not significantly different from one another. However, when the data were analyzed on the basis of whether or not the hypothyroid mothers had been treated during pregnancy, there was a striking change in the results. The full-scale mean IQs of children of the 124 controls and of the 14 treated hypothyroid mothers were 107 and 111 respectively, which were significantly different $(P=0.005)$ from the mean IQ of 100 of the children from the 48 untreated hypothyroid mothers.

Further examination showed that 33\% of children from the 48 untreated mothers had IQs 1 s.D. below the control mean, compared with $15 \%$ of the control children and only $7 \%$ of the children from the 14 treated mothers. Four times as many of the children from the 48 untreated hypothyroid mothers had IQs 2 s.D. below the control mean, as did children from the 124 control mothers. Interestingly, none of the children of the treated hypothyroid women had IQs 2 s.D. below the control mean. Additional studies have demonstrated a significant inverse correlation between maternal TSH and IQ of the offspring (7).

A comparison of the mean hormonal values of the treated and untreated mothers at 17 weeks showed no significant difference in any of the biochemical markers. If anything, the mean TSH of $17.8 \mathrm{mu} / \mathrm{l}$ of the treated group was insignificantly higher than the mean TSH of $12.1 \mathrm{mU} / \mathrm{l}$ of the untreated mothers. The abnormally low $\mathrm{FT}_{4}$ of $9.0 \mathrm{pmol} / \mathrm{l}$ was the same in both cohorts. In view of these data, we surmised that the circulating level of thyroid hormone had been normal in the treated cohort of mothers at a 
critical juncture before 17 weeks' gestation. At some later point, however, the $\mathrm{T}_{4}$ replacement dosage had become inadequate because of the increasing concentration of thyroxine binding globulin (TBG) and the heightened demands of progressing pregnancy.

\section{Intervention}

Although the precise time for intervention with thyroxine to prevent brain damage in the offspring is unknown, there are limited data that are helpful. Liu et al. reported eight mothers who were diagnosed and treated for autoimmune hypothyroidism at 5-10 weeks of gestation. Their circulating thyroxine levels became normal by 13-28 weeks of gestation. All the children had normal IQs when tested at 4-10 years of age (8). Radetti et al. identified eight pregnant women with subclinical and one woman with overt autoimmune hypothyroidism at weeks $8-10$ of gestation. They were started on thyroxine soon after the diagnosis was made. Their children had normal developmental tests at 9 months of age (9). The recent report by Pop et al. (10) describes infant neurodevelopment at 2 years of age in 57 children whose mothers were hypothyroxinemic at 12 weeks' gestation. The authors found that the $\mathrm{FT}_{4}$ concentrations increased spontaneously in $26 \%$ of the women during the first half of gestation. Their offspring had normal IQs compared with the low IQs of children from the women whose $\mathrm{FT}_{4}$ remained below the 10th percentile during the duration of the pregnancy.

While data from these studies are encouraging, it will require much more information from women treated at different times throughout the first half of gestation before we can draw any valid conclusions regarding optimal time of treatment. In the meantime, every effort should be made to educate both patients and caretakers on the importance of diagnosing and treating gestational subclinical hypothyroidism at the earliest possible time. Unless action is taken to test every pregnant woman as early in pregnancy as possible (or even before conception), society will continue to pay a heavy price in the number of damaged offspring. The magnitude of the problem can be more readily appreciated by examining the effects of congenital hypothyroidism on infants before newborn screening programs were implemented.

\section{Benefits of screening}

In several well-documented studies, summarized in a monograph (11), the incidence of clinically diagnosable congenital hypothyroidism was approximately 1:6500 births. Since $65 \%$ of the afflicted infants had an IQ 1 s.D. below the control mean, this translated to one damaged infant per 10000 full-term births.
In comparison, $38 \%$ of children from mothers whose TSH was at or above the 99.7 percentile had IQs 1 S.D. below the control mean. This results in 11 children per 10000 live births with IQs 1 S.D. below the control mean (6). We are left with the grim statistic that at least 11 times as many children born to mothers with gestational hypothyroidism will have IQs 1 S.D. below the control mean as hypothyroid infants before screening.

In view of these sobering facts, it should be the responsibility of the medical community to outline a course of action that will bring relief to pregnant hypothyroid women and their unborn children. Considering the current climate of fiscal constraint, it is our opinion that the most practical approach is to screen all pregnant women for hypothyroidism as early in pregnancy as possible (or before conception). We believe that screening programs modeled after those designed for congenital hypothyroidism would prove to be cost-effective and beneficial for both mother and child.

In the case of the mother, screening would result in early diagnosis and treatment of subclinical hypothyroidism. Unfortunately, pregnant women with subclinical hypothyroidism seem to escape early clinical detection. In our earlier study, $58 \%$ of the hypothyroid women were unaware of their disorder, and it took a median of 5 years from the time of the pregnancy for the clinical diagnosis to be made (6). The implications are staggering when one considers that there is a significant increase in intrauterine deaths, spontaneous abortions, premature births and pre-eclampsia in women with gestational subclinical hypothyroidism.

In the infant, major malformations and loss of IQ could be prevented by early diagnosis and treatment of the mother. Screening could be done most economically and expeditiously by using filter-paper blood specimens obtained at the first prenatal visit (or before conception). The specimens could be forwarded by mail or courier to the regional newborn-screening laboratory for $\mathrm{TSH}$ and $\mathrm{T}_{4}$ determinations. In iodidedeficient areas, thyroglobulin might be a better marker than TSH, but not in iodine-sufficient areas (12). In any event, treatment should be designed to maintain $\mathrm{FT}_{4}$ in the upper half of the normal reference range and TSH in the lower half of the normal reference range.

In the USA, a regional screening laboratory charges approximately $\$ 5$ to measure TSH and $\mathrm{T}_{4}$, supply the filter paper and report the results. Since approximately 1:1000 newborns is at risk of brain damage due to maternal subclinical hypothyroidism, the cost of identifying a child at risk is approximately $\$ 5000$. Clearly, this is a cost-effective approach.

We believe that if screening of all pregnant women is implemented, the mother, the infant and society will all benefit. 


\section{References}

1 Morreale de Escobar G, Obregon MJ \& Escobar del Rey F. Fetal and maternal thyroid hormones. Hormone Research 198726 12-27.

2 Vulsma T, Gons MH \& de Vijlder JJM. Maternal-fetal transfer of thyroxine in congenital hypothyroidism due to a total organification defect or thyroid agenesis. New England Journal of Medicine $198932113-16$

3 Man EB \& Jones WS. Thyroid function in human pregnancy. $\mathrm{V}$. Incidence of maternal serum low butanol-extractable iodines and of normal gestational TBG and TPBA capacities: retardation of 8-month-old infants. American Journal of Obstetrics and Gynecology 1969104 898-908.

4 Klein RZ, Haddow JE, Faix JD, Brown RS, Hermos RJ, Pulkkinen A \& Mitchell ML. Prevalence of thyroid deficiency in pregnant women. Clinical Endocrinology 199135 41-46.

5 Allan WC, Haddow JE, Palomaki GE, Williams JR, Mitchell ML, Hermos RJ, Faix JD \& Klein RZ. Maternal thyroid deficiency and pregnancy complications: implications for population screening. Journal of Medical Screening 20007 127-130.

6 Haddow JE, Palomaki GE, Allan WC, Williams JR, Knight GJ, Gagnon J. O'Heir CE, Mitchell ML, Hermos RJ. Waisbren SE Faix JD \& Klein RZ. Maternal thyroid deficiency during pregnancy and subsequent neuropsychological development of the child. New England Journal of Medicine 1999341 549-555.

7 Klein RZ, Sargent JD, Larsen PR, Waisbren SE, Haddow JE \& Mitchell ML. Relation of severity of maternal hypothyroidism to cognitive development of offspring. Journal of Medical Screening $2001818-20$.

8 Liu H, Momotani N, Noh JY, Ishikawa N, Takebe K \& Ito K. Maternal hypothyroidism during early pregnancy and intellectual development of the progeny. Archives of Internal Medicine 1994 $154785-787$.

9 Radetti G, Gentili L, Paganini C, Oberhofer R, Deluggi I \& Delucca A. Psychomotor and audiological assessment of infants born to mothers with subclinical thyroid dysfunction in early pregnancy. Minerva Pediatrics 200012 691-698.

10 Pop VJ, Brouwers EP, Vader HL, Vulsma T, van Baar AL \& deVijlder JJ. Maternal hypothyroxinaemia during early pregnancy and subsequent child development: a 3-year follow-up study. Clinical Endocrinology $2003 \mathbf{5 9} 282-288$.

11 Klein RZ. Infantile hypothyroidism then and now: the results of neonatal screening. Current Problems in Pediatrics 198515 5-56.

12 Mitchell ML, Klein RZ, Sargent JD, Meter RA, Haddow JE, Waisbren SE \& Faix JD. Iodine sufficiency and measurements of thyroid function in maternal hypothyroidism. Clinical Endocrinology $2003 \mathbf{5 8} 612-616$

Received 26 May 2004

Accepted 5 August 2004 